\title{
290. Ergebnisse der konservativen Behandlung von Unterschenkelschaftfrakturen
}

\author{
H. R. Siebert, M. Ebersberger und A. Pannike \\ Unfallchirurgische Klinik der Johann-Wolfgang-Goethe-Universität, \\ Theodor-Stern-Kai 7, D-6000 Frankfurt/M.
}

\section{Results of Conservative Treatment of Lower-Leg Fractures}

Summary. Of 235 fractures of the lower leg, $123(44 \%)$ were treated by closed reduction, extension for 3 weeks, and by splint. Follow-up after $2-3$ years showed a slow union rate of $12 \%$, no osteitis, no embolism of the pulmonary artery, and no pseudarthrosis. In $60 \%$ there was an unlimited range of active knee-joint motion, in $3 \%$ a limited range $\left(0^{\circ}-10^{\circ}-90^{\circ}\right)$. The rate of arthrosis (X-ray) of the knee joint $2-3$ years later was $6 \%$ in these patients. When these results are compared with those of operative treatment, there is no essential difference.

Key words: Conservative treatment - Lower-leg fractures - Late results.

Zusammenfassung. In der Zeit vom 1. 1 1975-31. 12. 1980 wurden an der Unfallchirurgischen Universitätsklinik Frankfurt/M, von 235 Unterschenkelschaftfrakturen $123(44 \%)$ konservativ behandelt. Das funktionelle Ergebnis 2-3 Jahre nach dem Unfall ergab eine freie Beweglichkeit im Kniegelenk und oberen Sprunggelenk bei $60 \%$ der Patienten, eine deutliche Bewegungseinschränkung im Kniegelenk von 3\%; im oberen Sprunggelenk von 12\%. Röntgenologische Arthroserate 2-3 Jahre nach dem Unfall im Kniegelenk $6 \%$ im oberen Sprunggelenk 4\%. Komplikationen : Lungenembolie $0 \%$, verzögerte Heilung $12 \%$ - alle heilten nach operativem Verfahren komplikationslos aus. Pseudarthrosen $0 \%$ irreversible Fibularisparese $3(2 \%)$.

Schlïsselwörter: Konservative Frakturenbehandlung - Unterschenkelschaftfraktur - Ergebnisse - Komplikationen.

\section{Gefahr der Nervenläsion bei Marknagelung der unteren Extremität}

\author{
W. Schlickewei, E. H. Kuner, V. Hendrich und R. Hoydem
}

Abteilung Unfallchirurgie (Ärztl. Dir.: Prof. Dr. E. H Kuner), Universitätsklinik Freiburg im Breisgau, Hugstetter Straße 55, D-7800 Freiburg/Br.

\section{The Risk of Nerve Lesion in Intramedullary Nailing of the Lower Extremity}

Summary. Nerve injuries in intramedullary nailing originate as a rule in intraoperative extension, postoperative positioning of the extremity, or in hematoma. Therefore, the lesion is usually neurapraxia or axonotmesis. Therapy should be conservative. In 287 cases of intramedullary nailing in a period of 4 years, we found 18 lesions of the peroneal nerve $(6.3 \%$, which were combined with tibial nerve lesions in 8 cases. At the control examination 3 years after injury, 3 patients were found to be handicapped in function. Therefore, the late complication rate was $1 \%$.

Key words: Intramedullary nailing - Nerve lesion.

Zusammenfassumg. Nervenverletzungen bei Marknagelungen entstehen in der Regel intraoperativ durch Dehnungsschäden bei Extension, Lagerungsfehler in der ersten postoperativen Phase oder durch postoperative Hämatombildung. Die Schädigung am Nerv ist deswegen zumeist eine Neurapraxie oder Axonotmesis. Die Therapie sollte konservativ sein. Bei 287 Marknagelungen in 4 Jahren hatten wir in unserem Patientengut insgesamt 18 postoperative Läsionen des Peronaeus $(6,3 \%)$, in 8 Fällen kombiniert mit dem N. tibialis. Bei Nachuntersuchung ca. 3 Jahre nach Verletzung waren noch 3 Patienten in ihrer Funktion behindert, was einer Spätkomplikationsrate von $1 \%$ entspricht.

Schliisselwörter: Marknagelung - Nervenverletzung. 